\title{
Adaptation of Moroccan Radiotherapy Services to the Covid-19 Outbreak
}

\author{
YOUBI Zakaria Ahmed ${ }^{1 *}$, LACHGAR Amine ${ }^{2}$, SELLAL Nabila ${ }^{1}$ and ELHFID Mohamed ${ }^{1}$ \\ ${ }^{1}$ Department of Radiation Therapy, Ahmad Bin Zayed Al Nahyan Center for Cancer Treatements, Morocco. \\ ${ }^{2}$ Department of Radiation Therapy, National Institute of Oncology, Morocco.
}

*Corresponding author: YOUBI Zakaria Ahmed, Radiation Therapy Department, Ahmad Bin Zayed Al Nahyan Center for Cancer Treatments, Tangier, Morocco.
Received Date: May 07, 2020

Published Date: May 22, 2020

\begin{abstract}
The pandemic of Corona virus affected almost all countries in the world and causing several thousand deaths. Africa in particular Morocco in full confinement to limit the damage has adopted several ways to protect its patients in anti cancer centers.
\end{abstract}

Keywords: Covid 19; Moroccan radiotherapy services; Outbreak

Abbreviations: COVID-19: coronavirus 2019; QRT-PCR: quantitative Reverse Transcription Polymerase Chain Reaction

\section{Commentary}

After WUHAN in China, the Coronavirus Disease 2019 pandemic has spread rapidly around the world, causing almost 117702 deaths to date [1]. Africa is also very affected by this pandemic with an ever-increasing curve of positive cases. Morocco among others, following very closely what was happening in other countries and particularly in Europe, has adopted a drastic proactive policy such as closing the borders, compulsory confinement and establishment of national protocols for standardized management of positive COVID-19 cases.

African countries are convinced that COVID-19 would have a huge impact on their health systems, which are already suffering due to the lack of medical resources and material (reanimation services, drugs, respirators, etc.) as well as the shortage of medical and paramedical personnel. As cancer patients are more vulnerable to infection compared to the general population, cancer centers in Africa, particularly in Morocco, will face many adaptative challenges and organizational issues imposed by this pandemic while ensuring continuity of care by balancing the risks-benefits. The radiotherapy services in Africa as well as the radiation oncologists will have the heavy task of rescheduling their protocols and the treatment circuit for their patients by adapting to current circumstances.

As no national guidelines are available so far, the measures taken in radiotherapy centers in Morocco follow the international recommendations for cancer care during COVID-19 crisis [2-7]. This includes delaying treatment of cancers with good prognosis and/ or slow progression, prioritizing curative radiotherapy over that used in palliative setting, imposing rigorous protection measures for both patients and medical staff, adopting hypo fractionated radiotherapy regimens validated by phase III studies and/or metaanalyzes, postponing post-therapeutic surveillance visits or, if necessary, shifting to telemedicine. All cancer cases are discussed in a multidisciplinary setting by web conferences in order to adopt the best therapeutic strategy with the minimum of risk. In light of the increasing burden of infections, some cancer centers will be dedicated to manage COVID-positive cancer patients with welltrained specialized doctors, nurses and appropriate reanimation 
services. Otherwise, these centers will be completely shifted to COVID-19 patients management but not cancer in worst-case scenarios.

These proactive and relevant measures altogether in addition to those carried out by the government (QRT-PCR Tests, Hospitals and special COVID 19 services, intensive care equipment, respirators, etc.) will enable our country to face this pandemic by circumventing the lack of local resources and maintaining continuity of care for our patients without exposing them to the risk of COVID-19 infection.

\section{Acknowledgment}

None

\section{Conflict of Interest}

No conflict of interest.

\section{References}

1. World Health Organization. Coronavirus disease (COVID-19) Pandemic.

2. Simcock R, Thomas TV, Estes C, Filippi AR, Katz MA, et al. (2020) COVID-19: Global radiation oncology's targeted response for pandemic preparedness. Clin Transl Radiat Oncol 22: 55-68.
3. Coles CE, Aristei C, Bliss J, Boersma L, Brunt AM, et al. (2020) International Guidelines on Radiation Therapy for Breast Cancer During the COVID-19 Pandemic. Clin Oncol 32(5): 279-281.

4. Zaorsky NG, Yu JB, McBride SM, Dess RT, Jackson WC, et al. (2020) Prostate Cancer Radiotherapy Recommendations in Response to COVID-19. Adv Radiat Oncol.

5. Braunstein LZ, Gillespie EF, Hong L, Xu A, Bakhoum SF, et al. (2020) Breast radiotherapy under COVID-19 pandemic resource constraintsapproaches to defer or shorten treatment from a Comprehensive Cancer Center in the United States. Adv Radiat Oncol.

6. Al Rashdan A, Roumeliotis M, Quirk S, Grendarova P, Phan T, et al. (2020) Adapting Radiotherapy Treatments for Breast Cancer Patients during the COVID-19 Pandemic: Hypo-Fractionation and Accelerated Partial Breast Irradiation to Address World Health Organization Recommendations. Adv Radiat Oncol.

7. COVID-19 rapid guideline: delivery of radiotherapy. NICE guideline [NG162]. 